\title{
Stability analysis of efficient solutions in multiobjective integer programming: A case study in load management
}

\author{
Maria João Alves $^{\mathrm{a}, \mathrm{c}, *}$, João Clímaco ${ }^{\mathrm{a}, \mathrm{c}}$, Carlos Henggeler Antunes ${ }^{\mathrm{b}, \mathrm{c}}$, Humberto Jorge ${ }^{\mathrm{b}, \mathrm{c}}$, \\ António G. Martins, ${ }^{b, c}$ \\ a Faculty of Economics, University of Coimbra, Av. Dias da Silva, 165, 3004-512 Coimbra, Portugal \\ ${ }^{\mathrm{b}}$ Department of Electrical Engineering and Computers, University of Coimbra, Polo II, 3030-290 Coimbra, Portugal \\ ' INESC Coimbra, Rua Antero de Quental, 199, 3000-037 Coimbra, Portugal
}

Available online 17 April 2006

\begin{abstract}
This paper presents a multiobjective linear integer programming model for supporting the choice of remote load control strategies in electric distribution network management. The model takes into account the main concerns in load management, considering three objective functions: minimization of the peak demand as perceived by the distribution network dispatch center, maximization of the utility profit associated with the energy services delivered by the controlled loads and minimization of the discomfort caused to consumers. The problem was analyzed using an interactive reference point method for multiobjective integer (and mixed-integer) linear programming. This approach exploits the use of the branch-and-bound algorithm for solving the reference point scalarizing programs through which efficient solutions are computed. Post-optimality techniques enable a stability analysis of the efficient solutions by means of computing and displaying graphically sets of reference points that correspond to the same solution.
\end{abstract}

(c) 2006 Elsevier Ltd. All rights reserved.

Keywords: Multiobjective integer programming; Interactive methods; Energy management

\section{Introduction}

Electric utilities have been using demand- and supply-side resources in an integrated manner, due to its potential attractiveness both at operational and economic levels. A growing interest is being devoted to these activities with the ongoing restructuring and liberalization trends in the energy sector, mainly due to the volatility and spikes of wholesale electricity prices and reliability concerns (transmission congestion and generation shortfalls). For instance, these programs can be very attractive for a retailer dealing with volatile wholesale prices and fixed, over a certain time period, retail prices.

The reduction of peak power demand is an important operational benefit associated with these activities [1-3].

The use of demand-side resources can be accomplished, in the framework of load management (LM), by means of the implementation of direct load control actions, consisting of power curtailment actions (on/off patterns) changing the regular working cycles of end-use loads. This entails changing the demand shape at more aggregate levels of loads.

\footnotetext{
* Corresponding author. Faculty of Economics, University of Coimbra, Av. Dias da Silva, 165, 3004-512 Coimbra, Portugal. Tel.: +351 239 790 500; fax: +351239790514 .

E-mail address: mjalves@fe.uc.pt (M.J. Alves).
} 
Those on/off patterns are generally applied, during a period of time, to groups of loads associated with energy services whose quality is not substantially affected by supply interruptions of short duration, such as electric water heaters and air conditioners in the residential sector. Since these are thermostatic loads, external changes to their working cycle have influence on their demand pattern in subsequent time periods. As a consequence, load management programs can also give rise to undesirable effects, such as the payback effect (whenever power is restored simultaneously to loads thus eventually creating another peak, when compared with the situation without load control actions) and possible reduction in revenues. This effect can be avoided by planning the on/off periods for all groups under control as non-coincident.

The selection of load control actions requires the explicit consideration of multiple, incommensurate and conflicting evaluation criteria of the merit of alternative load shedding strategies, capable of reflecting economic, technical and quality of service aspects. The quality of service dimension is crucial because consumers' acceptance is indispensable for the success of load management programs. A multiobjective linear integer model for supporting the choice of remote load control strategies in electric distribution network management has been developed. The model takes into account the main concerns of load management, considering three objective functions: minimization of peak demand as perceived by the distribution network dispatch center, maximization of utility profit corresponding to the energy services delivered by the controlled loads and minimization of discomfort caused to consumers.

In these complex combinatorial problems, several sources of uncertainty influence the quality of the data used to compute the coefficients of the mathematical model. Moreover, in presence of multiple evaluation aspects, operationalized by objective functions, the decision maker's preferences also play a crucial role in the selection of a final (compromise) nondominated solution to be implemented. Therefore, a methodological interactive approach is used, which enables to perform a (post-optimal) stability analysis of the nondominated solutions in face of changes of the decision maker's preferences. This method is devoted to multiobjective integer (and mixed-integer) linear programming problems and it is based on reference points (consisting of aspiration levels the decision maker would like to attain for each objective function). It exploits the use of the branch-and-bound algorithm for solving the reference point scalarizing programs through which nondominated solutions are computed, also displaying graphically sets of reference points that correspond to the same solution (thus avoiding an exhaustive search).

In this section, the interest and motivation of the study have been provided. In Section 2, the methodological approach to search for nondominated solutions and perform a stability analysis of the nondominated solutions is presented. The mathematical model for the selection of control strategies is presented in Section 3. The analysis of the problem is described in Section 4. In Section 5 some conclusions are drawn on the suitability of the proposed approach to analyze the stability of solutions to the load management problem.

\section{Overview of the methodological approach}

Let us consider the multiple objective integer linear programming (MOILP) problem:

$$
\begin{array}{ll}
\max & f_{1}(\boldsymbol{x})=c^{1} \boldsymbol{x} \\
\cdots & \\
\max & f_{k}(\boldsymbol{x})=\boldsymbol{c}^{k} \boldsymbol{x} \\
\text { s.t. } & \boldsymbol{x} \in X=\{\boldsymbol{x} \mid \boldsymbol{A x}=\boldsymbol{b}, \boldsymbol{x} \geqslant \mathbf{0}, \boldsymbol{x} \text { integer }\}
\end{array}
$$

where $\boldsymbol{x}$ is an $n$-dimensional vector of variables, $\boldsymbol{A}$ is an $m \times n$ matrix, $\boldsymbol{b}$ is the RHS vector and the vectors $\boldsymbol{c}^{i}(i=1, \ldots, k)$ represent the coefficients of the objective functions (criteria). It is assumed that $X$ is bounded and $f_{i}(\boldsymbol{x})=\boldsymbol{c}^{i} \boldsymbol{x}(i=1 \ldots k)$ are also integer-valued for all feasible $\boldsymbol{x}$.

Let $Z$ denote the feasible region in the criteria space, i.e. the set of points $z \in \mathfrak{R}^{k}$ such that $z_{i}=f_{i}(\boldsymbol{x}), i=1, \ldots, k$, $x \in X$.

$\boldsymbol{x}^{\prime} \in X$ is an efficient or nondominated solution iff there is no $\boldsymbol{x} \in X$ such that $\boldsymbol{c}^{i} \boldsymbol{x} \geqslant \boldsymbol{c}^{i} \boldsymbol{x}^{\prime}$ for all $i$ and $\boldsymbol{c}^{j} \boldsymbol{x}>\boldsymbol{c}^{j} \boldsymbol{x}^{\prime}$ for at least one $j$.

$\boldsymbol{x}^{\prime \prime} \in X$ is said to be weakly efficient/nondominated iff there is no $\boldsymbol{x} \in X$ such that $\boldsymbol{c}^{i} \boldsymbol{x}>\boldsymbol{c}^{i} \boldsymbol{x}^{\prime \prime}$ for all $i$.

Although the term efficient is more often used for points $x$ and the term nondominated for points $z$, they can be used interchangeably.

MOILP problems and other multiobjective problems with non-convex feasible regions admit not only supported efficient solutions but also unsupported efficient solutions, i.e. solutions that do not belong to the frontier of the convex 
hull of the feasible region. These solutions cannot be obtained by optimizing scalar surrogate functions consisting in weighted-sums of the objective functions. For that reason, reference point scalarizing functions, which have been introduced by Bowman [4] and Wierzbicki [5], among others, are more adequate to deal with MOILP problems. Several developments on reference point approaches, namely interactive methods, have been further reported in the literature (e.g. [6-9]).

Let $\boldsymbol{q}$ denote a criterion reference point that may represent aspiration levels that the decision maker (DM) would like to attain for the objective functions. Let us consider the min-max scalarizing program $\min _{x \in X}\left\{\max _{i=1 \ldots k}\left(q_{i}-f_{i}(\boldsymbol{x})\right)\right\}$, which projects $\boldsymbol{q}$ onto the nondominated solution set. Since the optimal solution to this scalarizing program may be a weakly efficient solution only, then the term $-\rho \sum_{i=1}^{k} f_{i}(\boldsymbol{x})$ is usually added to ensure the efficiency condition, where $\rho>0$ is a small constant. This scalarizing program, which is

$$
\min _{x \in X}\left(\left\{\max _{i=1 \ldots k}\left(q_{i}-f_{i}(\boldsymbol{x})\right)\right\}-\rho \sum_{i=1}^{k} f_{i}(\boldsymbol{x})\right),
$$

is equivalent to (2):

$$
\begin{array}{ll}
\min & \left(\alpha-\rho \sum_{i=1}^{k} f_{i}(\boldsymbol{x})\right) \\
\text { s.t. } & f_{i}(\boldsymbol{x})+\alpha \geqslant q_{i}, \quad i=1, \ldots, k, \\
& \boldsymbol{x} \in X .
\end{array}
$$

If $\boldsymbol{q}>\boldsymbol{z}$ for all $z \in Z$, then the optimal solution to (2) is the nondominated solution to (1) closest to $\boldsymbol{q}$ according to the (augmented) Tchebycheff metric. Otherwise, (2) does not minimize a distance, but the result is also a nondominated solution. Actually, as stated by Wierzbicki [10], this minimization does not mean "coming close" in a traditional sense, but "coming close or better".

Alves and Clímaco [9] developed an interactive method for MOILP (and mixed-integer) programs, which uses the scalarizing program (2) to compute efficient solutions. The method is mainly devoted to perform directional searches by solving scalarizing programs (2) parameterized on $\boldsymbol{q}$.

Basically, this interactive method works as follows. At each interaction the DM can assess directly a new reference point $(\boldsymbol{q})$, which is inserted into the scalarizing program (2), or just select an objective function he wants to improve regarding the efficient solution previously computed. In the former case, the result is a new efficient solution or an already known solution, since there are multiple reference points that lead to the same solution. In the latter case, the DM aims at performing a directional search and the procedure ensures that a solution which is different from the previous one is obtained, because the reference point is automatically adjusted. This stage involves an iterative process of sensitivity analysis based on the branch-and-bound algorithm.

Let us consider that $\overline{\boldsymbol{q}}$ is the reference point used in the previous computation of a directional search, which led to the efficient solution $\overline{\boldsymbol{x}}$. For short, we say that the reference point $\overline{\boldsymbol{q}}$ leads to the efficient solution $\overline{\boldsymbol{x}}$ if $\overline{\boldsymbol{x}}$ optimizes the scalarizing program (2) with the vector of parameters $\overline{\boldsymbol{q}}$. Besides, let us consider that the DM selected the objective function $f_{p}$ to be improved. Hence, the iterative sensitivity analysis procedure determines $\bar{\theta}_{p} \geqslant 0$ such that reference points between $\overline{\boldsymbol{q}}=\left(\bar{q}_{1}, \ldots, \bar{q}_{k}\right)$ and $\left(\bar{q}_{1}, \ldots, \bar{q}_{p}+\bar{\theta}_{p}, \ldots, \bar{q}_{k}\right)$ still lead to $\overline{\boldsymbol{x}}$, but for $\theta_{p}>\bar{\theta}_{p}$ a different efficient solution is obtained. The sensitivity analysis procedure uses information provided by the branch-and-bound tree that solved the integer program (2) for the reference point $\overline{\boldsymbol{q}}$. The tree structure and some information on the terminal nodes are preserved between consecutive computations. They are used to determine $\bar{\theta}_{p}$, also providing a starting structure for computing the new efficient solution that optimizes (2) for the new reference point, i.e., $\hat{\boldsymbol{q}}=\left(\bar{q}_{1}, \ldots, \bar{q}_{p}+\hat{\theta}_{p}, \ldots, \bar{q}_{k}\right)$ with $\hat{\theta}_{p}$ slightly over $\bar{\theta}_{p}$. The previous branch-and-bound tree is firstly simplified by cutting branches corresponding to variable constraints that are no longer active. The tree is then expanded if new branching is required until the new optimal solution for the scalarizing program is reached, which improves the objective function $f_{p}$ selected by the DM. This procedure enables to save time in the computational phases promoting the directional searches for efficient solutions. Besides choosing the objective to be improved at each moment, the DM has also the possibility of imposing constraints on the objective function values in order to have more control of the directional searches. These constraints (of type $f_{i}(\boldsymbol{x}) \geqslant l_{i}$ for one or more $i \in\{1, \ldots, k\}$ ) may be revised whenever the DM wants, by relaxing or tightening the bounds. If a local analysis is made (which may be useful in a final phase of the decision process) the DM can easily 
conclude whether a region of interest is completely explored or not. More details about this interactive MOILP method can be found in [9].

Throughout a directional search for improving $f_{p}$ the procedure identifies reference points from $\overline{\boldsymbol{q}}$ to $\left(\bar{q}_{1}, \ldots, \bar{q}_{p}+\right.$ $\bar{\theta}_{p}, \ldots, \bar{q}_{k}$ ) that lead to the same efficient solution, say $\bar{x}$. Therefore, it is indifferent to consider any of those reference points in the scalarizing program to obtain the efficient solution $\overline{\boldsymbol{x}}$. This means that there is an indifference or stability set of reference points associated with each efficient solution.

Besides points between $\overline{\boldsymbol{q}}$ and $\left(\bar{q}_{1}, \ldots, \bar{q}_{p}+\bar{\theta}_{p}, \ldots, \bar{q}_{k}\right)$, other reference points belonging to the stability set of $\overline{\boldsymbol{x}}$ can easily be identified. Let $s$ denote the vector of surplus variables corresponding to the first $k$ constraints of (2), and $\bar{s}$ their optimal values when $\boldsymbol{q}=\overline{\boldsymbol{q}}$. Hence, all the reference points $\left(\bar{q}_{1}+\delta_{1}, \ldots, \bar{q}_{p}+\delta_{p}, \ldots, \bar{q}_{k}+\delta_{k}\right)$ with $0 \leqslant \delta_{i} \leqslant \bar{s}_{i}$ $(i=1, \ldots, k)$ belong to the stability set of $\overline{\boldsymbol{x}}$. Note, however, that at least one $\bar{s}_{i}=0$ according to the mathematical properties of the min-max problem (2), as $\alpha=\max _{i=1 \ldots k}\left(q_{i}-f_{i}(x)\right)$. Combining the information on the reference points obtained throughout directional searches with this simple analysis, the procedure is able to compute one or more convex sets of reference points that lead to $\overline{\boldsymbol{x}}$. The whole stability set of a given solution is usually non-convex, and difficult to determine. In general, this procedure is not able to define it completely, but just characterize some of its convex sub-sets [11].

For MOILP problems with 2 or 3 objective functions those stability sets (regions) can be graphically visualized. Let us focus on the three-objective case, which concerns our case study. The 3D stability regions of a three-objective problem can be represented on a $2 \mathrm{D}$ graph by considering the cut $q_{1}+q_{2}+q_{3}=\mathrm{S}$, with $\mathrm{S}$ constant. Since the optimal solution to (2) does not change if a constant is added to all components of $\boldsymbol{q}$, each stability region can be represented on the plane defined by $q_{1}+q_{2}+q_{3}=\mathrm{S}$ without loss of information. If the origin of the graph is adequately chosen (ensuring that any stability region has a representation on this graph) and considering, for instance $q_{1}$ on the $\mathrm{x}$-axis and $q_{2}$ on the $\mathrm{y}$-axis, the graph takes on a triangular shape. In this representation, $q_{3}=\mathrm{S}-q_{1}-q_{2}$. More details about the definition and graphical representation of these regions can be found in [11].

The interpretation of stability regions provides important insights into the MOILP problem because they help the DM (or the procedure) to avoid the selection of reference points that lead to the same efficient solution. Besides, they can induce a better perception of some characteristics of the efficient solution set, such as the number of solutions and their closeness in the objective function space. In sum, this information enables a stability analysis for each efficient solution with respect to the change of the reference point, also providing stability degrees (through the areas of the stability regions) for the efficient solutions with respect to the improvement of an objective function. Note that, in the framework of an interactive decision aid procedure, the DM is required to provide preference information in the objective function space (levels he would like to attain for each objective, and/or objective to improve). This is the space he is most familiar with and, therefore, this information does not involve an excessive burden (such as marginal rates of substitution or other preference information requirements).

The methodology presented in this section has been applied to a load management case study, whose model is presented in the next section.

\section{The load management problem}

The model presented herein is a slightly changed version of the model previously proposed by Jorge et al. [12], taking into account evaluation aspects of different nature (technical, economical and quality of service) which are at stake in load management policies. The three objective functions, which operationalize these evaluation aspects in our model, are: minimize the peak demand as perceived by the distribution network dispatch center, maximize the utility profit associated with the energy services delivered by the controlled loads, and minimize the discomfort that is caused to consumers by control actions. The use of this multiobjective model allows the DM to select a control strategy to apply to each group of controlled loads, in order to guarantee a compromise efficient solution among the three objectives.

The model does not depend on the type of controlled load, though it has been applied only to the control of electric water heaters groups. The information on the electricity consumption of the load groups and on hot water temperatures with or without the application of the control strategies to the load groups is obtained with a physically based load model [13]. Control strategies define the on/off schedule of load groups, during the period of time where maximum demand control is to be achieved. 


\subsection{Mathematical model}

The network load diagram is assumed to be known by means of a load forecast procedure. The total control period $(\Delta T)$, which must be as long as needed in order to prevent a new peak demand caused by the payback phenomenon, is divided into $n$ equal intervals $(\Delta t=\Delta T / n)$. In the following, let $i$ denote the elementary time interval index $(i=1, \ldots, n)$, $j$ the load group index $(j=1, \ldots, m)$ and $k$ the control strategy index $(k=1, \ldots, v) ; x_{j k}$ is the binary decision variable that assumes the value 1 if control strategy $k$ is selected to be applied to group $j$, and 0 otherwise $(j=1, \ldots, m$; $k=1, \ldots, v)$.

Let us first define the objective functions.

Under load control, the average network demand $\left(X_{i}\right)$ at each elementary interval $i$ is given by

$$
X_{i}=\sum_{j} \sum_{k}\left(c_{i j k} x_{j k}\right)+L_{i}, \quad i=1, \ldots, n,
$$

where $c_{i j k}$ is the difference at interval $i$ between the demand of load group $j$ when control strategy $k$ is applied to it and its demand without any control action; $L_{i}$ is the average forecasted network demand at interval $i$ without load control.

The first objective consists in minimizing peak demand, or, equivalently, in minimizing the maximum network controlled demand, that is: $\min \max \left\{X_{1}, X_{2}, \ldots, X_{n}\right\}$. This objective can be formulated in an alternative way, maximizing the peak demand reduction: $\max \min \left\{P-X_{1}, P-X_{2}, \ldots, P-X_{n}\right\}$, where $P$ is the forecasted network peak demand without control.

This objective is not linear, but it can be transformed into a linear one, solving the following linear problem:

$$
\max F_{1}=r
$$

s.t.

$$
P-X_{i}-r \geqslant 0 \Leftrightarrow P-\left(\sum_{j} \sum_{k}\left(c_{i j k} x_{j k}\right)+L_{i}\right)-r \geqslant 0 \quad(i=1, \ldots, n), r \geqslant 0,
$$

where $r$ is the variable that represents the network peak demand reduction.

Hence, (4) is the first objective function and (5) is a set of constraints for the multiobjective problem.

The second objective consists in optimizing the profit. This is equivalent to maximizing the revenue variation caused by the electricity consumption variation achieved with the application of control strategies. Thus, maximizing profit can be stated as

$$
\max \quad F_{2}=\sum_{j} \sum_{k}\left(R_{j k} x_{j k}\right),
$$

where $R_{j k}=(\Delta t / 60) \sum_{i} c_{i j k} m_{i j}$ is the profit variation corresponding to the consumption variation in group $j$ when subject to control strategy $k, m_{i j}$ being the net revenue perceived by the utility per KWh at interval $i$ by selling energy to group $j$.

The third objective consists in minimizing the consumers' discomfort. The measurement of the discomfort caused by control actions is based on the number of loads for which the minimum comfort threshold has been violated.

Minimizing the discomfort caused to consumers corresponds to minimizing the following function:

$$
\min \quad F_{3}=\sum_{j} \sum_{k} D_{j k} x_{j k},
$$

where $D_{j k}=\alpha_{A} A_{j k}+\alpha_{B} B_{j k} ; \alpha_{A}$ and $\alpha_{B}$ are coefficients of importance with respect to the accumulated value $\left(A_{j k}\right)$ and the maximum number $\left(B_{j k}\right)$ of loads in group $j$ that violate the minimum comfort threshold, when subject to control strategy $k$, and $D_{j k}$ is an aggregate measure of discomfort defined as a function of $A_{j k}$ and $B_{j k}$.

Besides (5), the other constraints of the model are the following:

(a) One control strategy should be applied to each load group

$$
\sum_{k} x_{j k}=1 \quad(j=1, \ldots, m)
$$


(b) There is a maximum number of loads that are allowed to violate the minimum comfort threshold. Control strategies that lead to a higher number of loads in such situation are rejected

$$
\sum_{k} B_{j k} x_{j k} \leqslant b_{j} \quad(j=1, \ldots, m),
$$

where $b_{j}$ is the maximum number of loads in group $j$ that are allowed to simultaneously violate the minimum comfort threshold.

(c) Decision variables $x_{j k}$ are binary

$$
x_{j k}=0 \text { or } 1 \quad(j=1, \ldots, m ; k=1, \ldots, v) .
$$

\section{Application of the interactive approach to the load management model}

Jorge et al. [12] used the well-known STEM method [14] to analyze a case study using the three-objective model described above. This case study corresponds to 4000 water heaters aggregated into eight groups. Eight control strategies were generated for each load group. The problem includes 65 variables ( 64 binary variables $x_{j k}$ plus variable $r$ ). The data used to supply the model have been collected from a Portuguese distribution company.

In the study presented in [12], the payoff table (displaying the objective function values for the solutions that optimize individually each objective function) plus five other efficient solutions were computed. Those solutions seem to provide interesting strategies, namely the last solution computed, which was considered by the DM a satisfactory compromise solution. However, due to the limitations of the STEM method it was not possible to make a comprehensive search for the efficient solution set. This is strongly recommendable namely because the a priori points of view of the electricity supply company and the points of view of the consumers are not compatible. Therefore, the multicriteria interactive analysis should contribute to an indispensable learning process putting in evidence not only all the relevant profiles of well contrasted nondominated alternative options but also providing aids to achieve a satisfactory compromise solution for the various actors. In the STEM method, the DM specifies a relaxation quantity for one objective function, in each interaction, in order to improve the other objectives. However, the DM has not the possibility of selecting an objective he would like to be privileged in the following computation(s). Instead, by following directional searches, the DM can choose the objective to be improved at each moment, having also the possibility of imposing constraints on the objective values.

In the next paragraphs, we describe the analysis of this load management model using the interactive reference point method (implemented in a software package) described in Section 2. The results are compared with those obtained using the STEM method, for the sake of illustration of the value-added resulting from the use of a decision aid tool capable of characterizing in a progressive and selective manner the nondominated solution set using the preference information provided by the DM as a guidance. Moreover, by knowing the stability regions associated with each nondominated solution, the DM avoids to specify preference information that would incur in a useless computational effort, for it would lead to solutions already known.

The MOILP software package started by computing the three efficient solutions that optimize individually each objective function, presented in Table 1, and the corresponding stability regions-Fig. 1. The stability regions displayed in Fig. 1 represent the loci of reference points that lead to the same efficient solution (in Fig. 1, the solutions that optimize individually each objective function) when computing efficient solutions using the scalarizing program (2).

It should be noted that the graphical representation of stability regions in Fig. 1 considers the system of points $\tilde{\boldsymbol{q}}=\left(\tilde{q}_{1}, \tilde{q}_{2}, \tilde{q}_{3}\right)=\left(q_{1} \times 10, q_{2},-q_{3}\right)$ due to: (a) $F_{3}$ is a minimizing function and $F_{1}$ and $F_{2}$ are maximizing functions,

Table 1

\begin{tabular}{|c|c|c|c|c|c|c|c|c|c|c|c|}
\hline \multirow[b]{2}{*}{ Solution 1} & \multirow{2}{*}{$\begin{array}{l}\operatorname{Max} F_{1}(\mathrm{KW}) \\
514\end{array}$} & \multirow{2}{*}{$\begin{array}{c}\operatorname{Max} F_{2}(\mathrm{PTE}) \\
8108\end{array}$} & \multirow{2}{*}{$\frac{\operatorname{Min} F_{3}}{4420}$} & \multicolumn{8}{|c|}{ Control strategies } \\
\hline & & & & 1 & 2 & 8 & 3 & 2 & 2 & 2 & 2 \\
\hline Solution 2 & 293 & 15402 & 7360 & 8 & 8 & 8 & 8 & 8 & 8 & 8 & 8 \\
\hline Solution 3 & 17 & 2976 & 980 & 6 & 3 & 3 & 6 & 6 & 3 & 6 & 7 \\
\hline
\end{tabular}

Solutions that optimize individually each objective function 


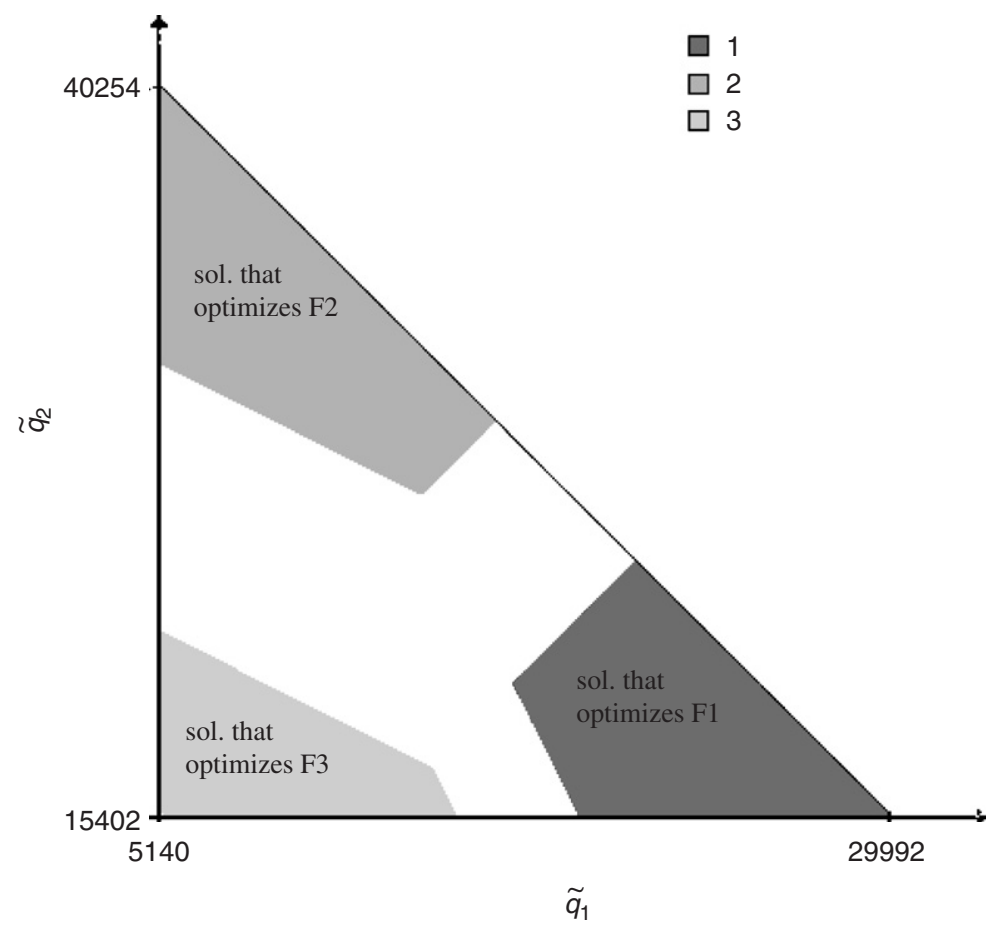

Fig. 1. Indifference regions for the solutions that optimize individually each objective function.

so the symmetric of $F_{3}$ is considered for scalarizing purposes; (b) $F_{1}$ has been changed to $F_{1} \times 10$ to measure the three objective function values on comparable orders of magnitude. Hence, an original reference point $\left(q_{1}, q_{2}, q_{3}\right)$ is converted into $\left(\tilde{q}_{1}, \tilde{q}_{2}, \tilde{q}_{3}\right)$ to be used in the scalarizing program (2) and also in the representation of the stability regions of the efficient solutions with respect to reference points.

In Table 1 (and the following), the Control Strategies are identified by a number that represents the control strategy applied to each group (from 1 to 8). For instance, the solution that optimizes $F_{1}$ (the one that most reduces the peak demand) consists in applying strategy 1 to group 1, strategy 2 to group 2, strategy 8 to group 3 , strategy 3 to group 4 , and so on.

Solutions 1, 2 and 3 give rise to the network load diagrams displayed in Figs. 2(a)-(c), respectively. The load curve corresponding to the situation without remote control actions is also displayed in each graph of Fig. 2.

After computing the first three solutions, a directional search was performed starting from solution 2 and choosing $F_{3}$ (the consumers' discomfort) to be improved since this was a very sacrificed criterion in solution 2 . Twenty-eight efficient solutions (from 4 to 31) were computed throughout this directional search. These solutions are shown in Table 2. Then, we decided to change the improvement direction because most of these solutions present very low values of reduction on the peak demand $\left(F_{1}\right)$.

Hence, the search was redirected to improve $F_{1}$ (starting from solution 31), and the new solutions from 32 to 36 were computed (see Table 3). Solution 36 is identical to the second solution obtained by Jorge et al. [12], thus also denoted as solution STEM-2: $\left(F_{1}, F_{2}, F_{3}\right)=(364,9265,2450)$.

At this stage we considered that $F_{1}$ had a reasonable value. Besides, $F 1$ values that satisfy $F_{1} \geqslant 300 \mathrm{KW}$ would also be acceptable. Therefore, this constraint was imposed and a new search was performed in order to improve profits $\left(F_{2}\right)$. Solution 37 was computed and new solutions-from 38 to 41 -followed it (see Table 3 ). As expected, all of them present a peak reduction of at least $300 \mathrm{KW}$ and growing values on profits. Solution 39 coincides with solution STEM-5 [12]. As this had been considered a satisfactory compromise solution in that study, we decided to make a local analysis around this one and the last solutions obtained.

The following bounds were then imposed: $F_{3} \leqslant 3000$ (which is not satisfied by solution 41 ) and $F_{2} \geqslant 10200$, keeping up $F_{1} \geqslant 300$. The nondominated solution closest to the last reference point (which led to solution 41) that satisfies these additional constraints is solution $40(300,10288,2930)$, already known. 

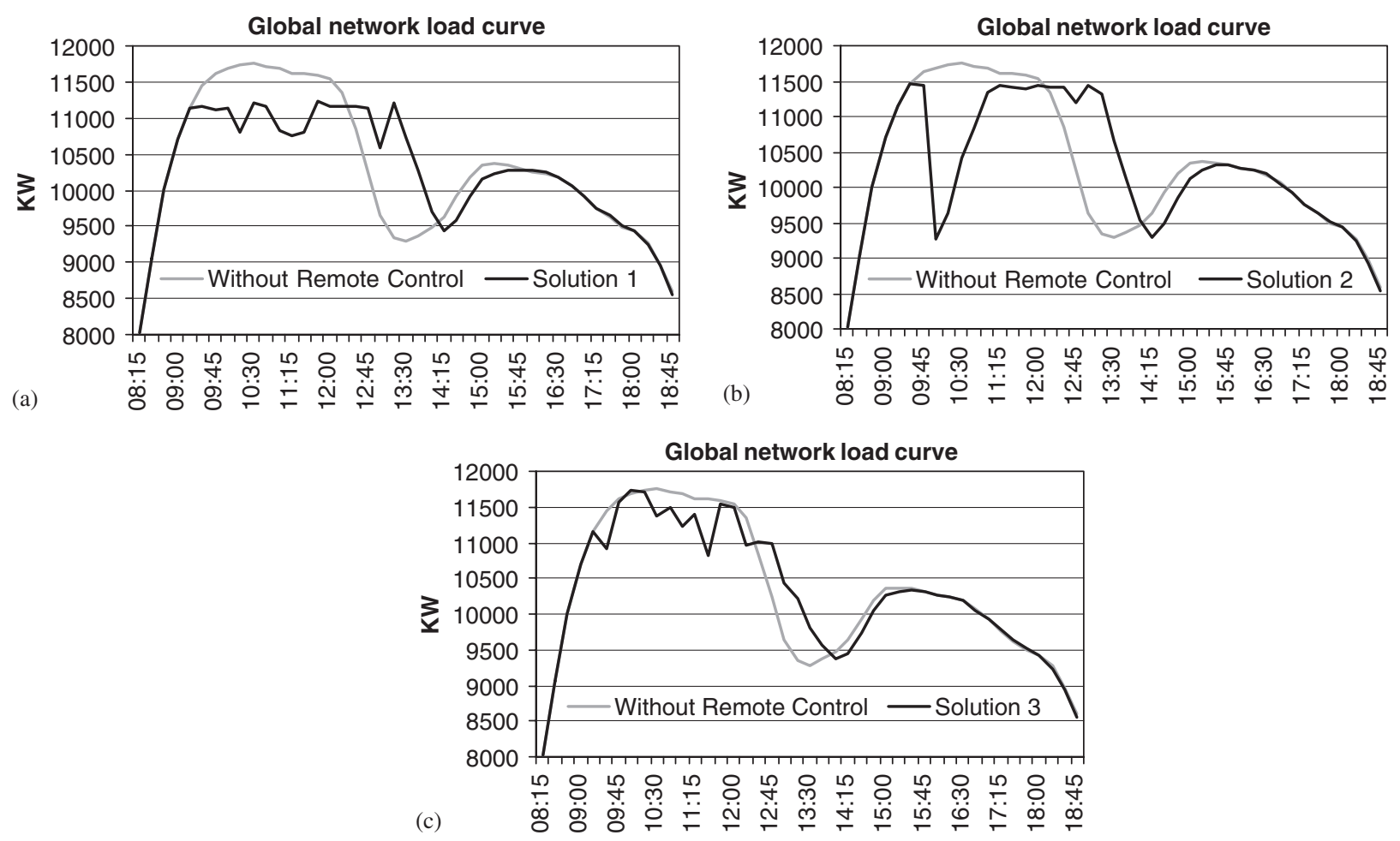

Fig. 2. Global network load curves corresponding to solutions 1,2 and 3.

The improvement of $F_{1}$, satisfying the same constraints, resulted in solution 39. Fig. 3 shows bar graphs for the last three solutions presented by the decision aid tool to the DM (41, 40 and 39, respectively) and the numerical information of solution 39.

Relaxing the bound on $F_{2}$ to $F_{2} \geqslant 10000$, and keeping the other bounds, the outcome for the same reference point is also solution 39. Moreover, if $F_{1}$ is chosen to be improved from this point, and maintaining the previous bounds, the procedure announces that no better value for $F_{1}$ is possible. Identical message is shown if $F_{3}$ is chosen to be improved. Therefore, this region of nondominated solutions was completely explored.

Fig. 4 shows the global network load curves corresponding to solutions 39 and 41, which are not very different (as it was expected). The load curves for the load groups under control in the same solutions (39 and 41) are displayed in Fig. 5.

Fig. 6 displays the stability regions for the 41 efficient solutions computed. It can be observed that there are cases where large variations of the reference point lead to the same efficient solution and other cases where a small change of the reference point is sufficient to result in a different efficient solution. Fig. 6 also presents the directions of improvement for each objective function. Hence, the stability regions indicate how stable an efficient solution is with respect to the DM's preferences, namely regarding the intention of improving a given objective function.

The analysis of the load management problem presented herein is just an example of a sequence of directional searches that could be followed. Since the software enables a free exploration of the problem, many other search strategies can be performed, providing more information about the problem. We made a deeper analysis and we realized that the problem has more than 200 efficient solutions. In fact, a large number of solutions should be expected by observing the large unfilled area on the graph in Fig. 6. As a curiosity, we can mention that among those 200 solutions only 39 are supported efficient solutions (solutions that can be obtained through the optimization of a scalar function consisting in a weighted-sum of the objective functions). In what concerns the search for the 41 solutions described above, 12 of them are supported and 29 are unsupported solutions.

From the analysis of this problem we can conclude that the successive efficient solutions obtained throughout directional searches, as well as the analysis of their stability regions, can give a good perception of the geometry of 
Table 2

Solutions computed in the first directional search

\begin{tabular}{|c|c|c|c|c|c|c|c|c|c|c|c|}
\hline & $\operatorname{Max} F_{1}(\mathrm{KW})$ & $\operatorname{Max} F_{2}(\mathrm{PTE})$ & $\operatorname{Min} F_{3}$ & \multicolumn{8}{|c|}{ Control strategies } \\
\hline \multicolumn{12}{|c|}{ Directional search starting from solution 2 to improve $F_{3}$ : } \\
\hline Solution 4 & 183 & 14652 & 7240 & 8 & 8 & 8 & 8 & 8 & 7 & 8 & 8 \\
\hline Solution 5 & 94 & 14294 & 6930 & 8 & 8 & 8 & 8 & 7 & 8 & 8 & 8 \\
\hline Solution 6 & 273 & 14281 & 6430 & 8 & 8 & 8 & 3 & 8 & 8 & 8 & 8 \\
\hline Solution 7 & 269 & 14034 & 5570 & 8 & 8 & 8 & 1 & 8 & 8 & 8 & 8 \\
\hline Solution 8 & 44 & 13778 & 5180 & 8 & 8 & 8 & 2 & 8 & 6 & 2 & 8 \\
\hline Solution 9 & 279 & 13371 & 5150 & 3 & 8 & 8 & 1 & 8 & 8 & 8 & 8 \\
\hline Solution 10 & 11 & 13228 & 4870 & 8 & 8 & 8 & 2 & 2 & 6 & 2 & 8 \\
\hline Solution 11 & 51 & 13133 & 4430 & 4 & 8 & 8 & 1 & 8 & 6 & 8 & 8 \\
\hline Solution 12 & 23 & 12947 & 3870 & 7 & 8 & 8 & 6 & 8 & 6 & 1 & 8 \\
\hline Solution 13 & 40 & 12784 & 3770 & 7 & 8 & 8 & 2 & 2 & 6 & 1 & 8 \\
\hline Solution 14 & 40 & 12372 & 3700 & 7 & 8 & 8 & 2 & 4 & 6 & 1 & 8 \\
\hline Solution 15 & 38 & 12175 & 3650 & 7 & 8 & 8 & 1 & 2 & 6 & 5 & 8 \\
\hline Solution 16 & 18 & 12141 & 3390 & 7 & 7 & 6 & 1 & 8 & 6 & 8 & 8 \\
\hline Solution 17 & 48 & 11866 & 3300 & 7 & 7 & 8 & 1 & 8 & 6 & 2 & 8 \\
\hline Solution 18 & 35 & 11732 & 2980 & 7 & 7 & 7 & 2 & 8 & 6 & 1 & 8 \\
\hline Solution 19 & 23 & 11355 & 2960 & 7 & 1 & 6 & 4 & 8 & 6 & 1 & 8 \\
\hline Solution 20 & 44 & 11345 & 2770 & 7 & 7 & 7 & 6 & 8 & 6 & 1 & 8 \\
\hline Solution 21 & 44 & 11196 & 2610 & 7 & 7 & 1 & 6 & 8 & 6 & 1 & 8 \\
\hline Solution 22 & 44 & 11033 & 2510 & 7 & 7 & 1 & 2 & 2 & 6 & 1 & 8 \\
\hline Solution 23 & 44 & 10621 & 2440 & 7 & 7 & 1 & 2 & 4 & 6 & 1 & 8 \\
\hline Solution 24 & 16 & 10463 & 2320 & 7 & 3 & 1 & 6 & 8 & 6 & 3 & 8 \\
\hline Solution 25 & 28 & 10371 & 2300 & 7 & 7 & 1 & 2 & 6 & 6 & 1 & 8 \\
\hline Solution 26 & 16 & 10140 & 2270 & 7 & 3 & 1 & 6 & 8 & 6 & 1 & 8 \\
\hline Solution 27 & 44 & 10088 & 2180 & 7 & 7 & 1 & 6 & 7 & 6 & 1 & 8 \\
\hline Solution 28 & 126 & 10069 & 2170 & 7 & 3 & 1 & 4 & 2 & 6 & 3 & 8 \\
\hline Solution 29 & 16 & 10068 & 2140 & 7 & 3 & 1 & 6 & 8 & 6 & 5 & 8 \\
\hline Solution 30 & 16 & 9977 & 2110 & 7 & 4 & 6 & 4 & 3 & 6 & 1 & 8 \\
\hline Solution 31 & 126 & 9770 & 1990 & 7 & 3 & 1 & 4 & 3 & 6 & 1 & 8 \\
\hline
\end{tabular}

Table 3

Solutions computed in the second and following directional searches

\begin{tabular}{|c|c|c|c|c|c|c|c|c|c|c|c|}
\hline & $\operatorname{Max} F_{1}(\mathrm{KW})$ & $\operatorname{Max} F_{2}(\mathrm{PTE})$ & $\operatorname{Min} F_{3}$ & \multicolumn{8}{|c|}{ Control strategies } \\
\hline \multicolumn{12}{|c|}{ Directional search starting from solution 31 to improve $F_{1}$ : } \\
\hline Solution 32 & 211 & 9644 & 2250 & 7 & 7 & 1 & 2 & 6 & 8 & 1 & 1 \\
\hline Solution 33 & 262 & 9776 & 2500 & 7 & 4 & 6 & 4 & 8 & 8 & 1 & 1 \\
\hline Solution 34 & 293 & 9497 & 2530 & 7 & 7 & 1 & 1 & 8 & 7 & 5 & 1 \\
\hline Solution 35 & 293 & 9679 & 2610 & 7 & 3 & 2 & 4 & 8 & 8 & 3 & 1 \\
\hline Sol. 36 (STEM-2) & 364 & 9265 & 2450 & 7 & 4 & 6 & 4 & 2 & 8 & 1 & 2 \\
\hline \multicolumn{12}{|c|}{ Changing direction to improve $F_{2}$, considering $F_{1} \geqslant 300 \mathrm{KW}$ : } \\
\hline Solution 37 & 331 & 9646 & 2690 & 7 & 7 & 7 & 1 & 8 & 7 & 5 & 1 \\
\hline Solution 38 & 319 & 9744 & 2760 & 7 & 7 & 7 & 2 & 2 & 7 & 1 & 2 \\
\hline Sol. 39 (STEM-5) & 319 & 10255 & 2810 & 7 & 7 & 7 & 2 & 8 & 7 & 1 & 1 \\
\hline Solution 40 & 300 & 10288 & 2960 & 7 & 7 & 7 & 2 & 8 & 8 & 4 & 1 \\
\hline Solution 41 & 362 & 10415 & 3130 & 7 & 7 & 7 & 1 & 8 & 6 & 5 & 2 \\
\hline
\end{tabular}

the efficient solution set. There exist "consecutive" solutions that present similar values for all the objective functions, but there are others that present very different values in one (or more) objective(s). An example of the first situation is the transition from solution 21 to 22 (see Table 2): $F_{1}$ maintains its value, $F_{2}$ diminishes $1.5 \%$ and $F_{3}$ improves $3.8 \%$. Thus, these solutions have close values for all the objective functions. An example of the second situation is the transition from solution 27 to 28 : $F_{1}$ improves $186 \%$ and $F_{3}$ improves $0.46 \%$, just sacrificing $F_{2}$ on $0.19 \%$. 


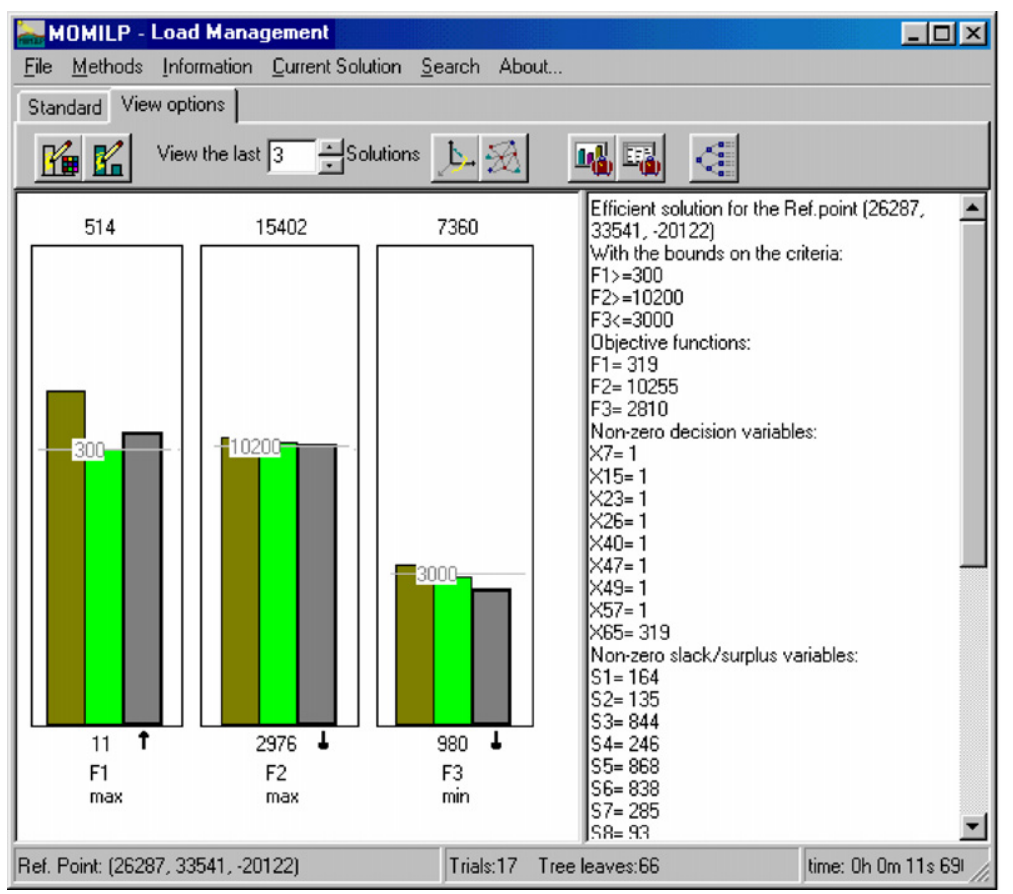

Fig. 3. Graphs of solutions 41, 40 and 39, respectively, and numerical information of solution 39.
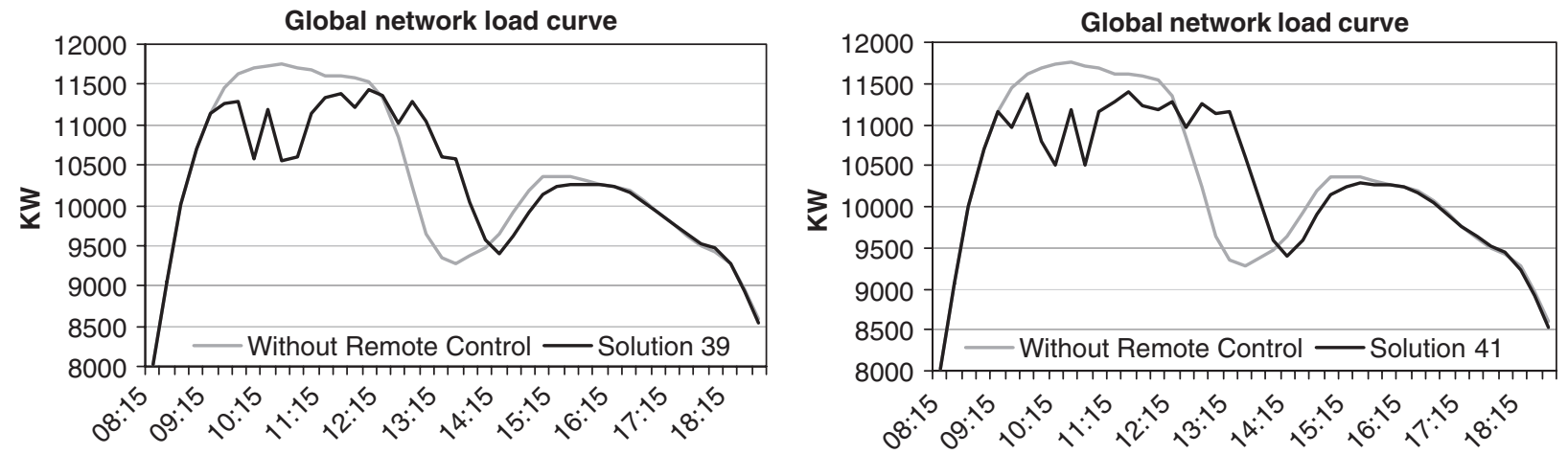

Fig. 4. Global network load curves corresponding to solutions 39 and 41.
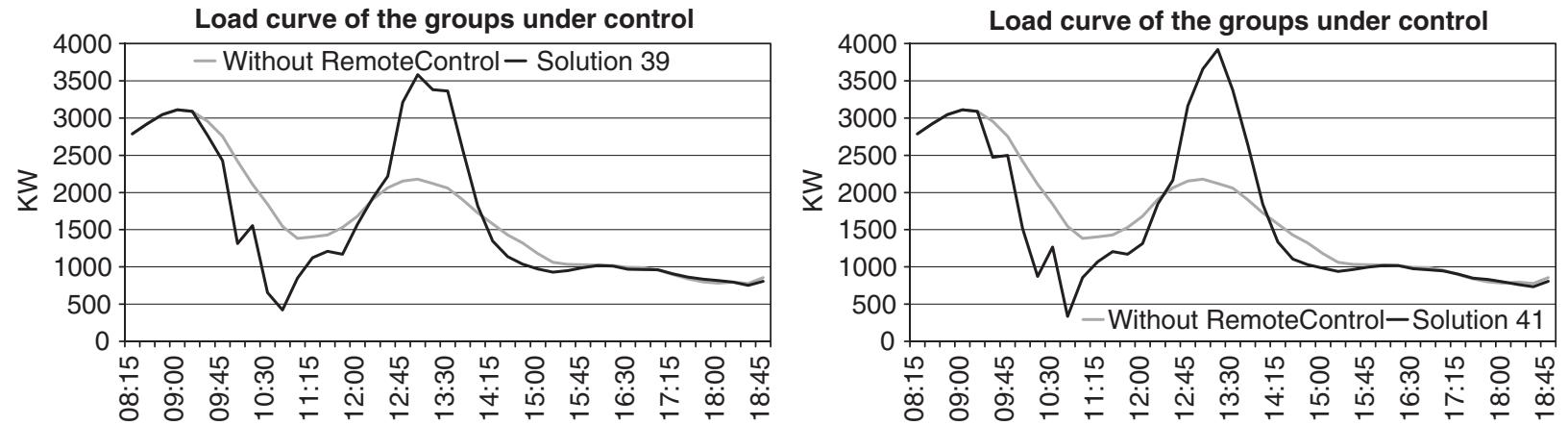

Fig. 5. Load curves of the groups under control in solutions 39 and 41. 


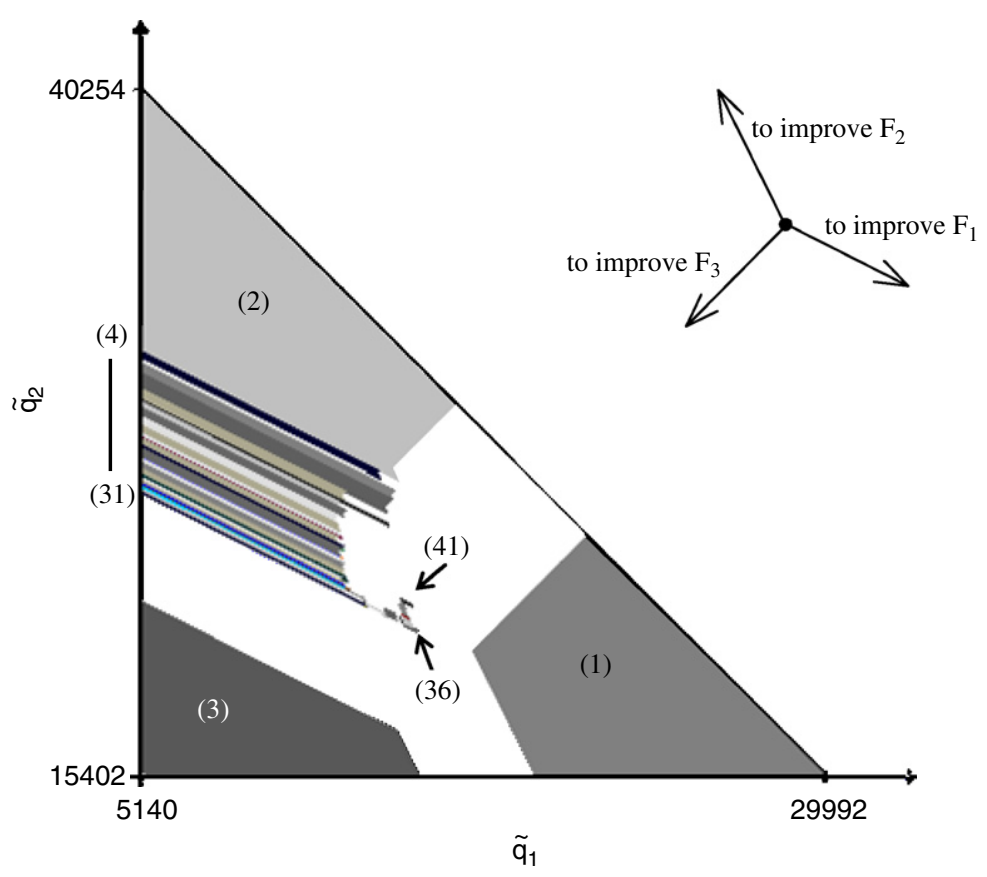

Fig. 6. Stability regions for all the efficient solutions computed.

These are "consecutive" solutions with similar values on $F_{2}$ and $F_{3}$, but a very different value on $F_{1}$. So, it can be easily considered that solution 28 is superior to solution 27.

\section{Conclusions}

In this paper we presented a study of a multiobjective load management problem using a decision support software having at its core an interactive reference point method for multiobjective (mixed) integer linear programming. The results have been compared with those obtained by the application of the STEM method to the same problem, for the sake of illustration of the value-added of the approach proposed herein.

The interactive reference point method used in this study is mainly devoted to perform directional searches. It also enables a stability analysis of the efficient solutions by providing a graphical representation of indifference reference points that result in the same solution (for problems up to three objective functions).

Several advantages of this approach are highlighted in this study of a load management problem:

- The procedure enables a free exploration of the decision alternatives, allowing for both a strategic search and a more local analysis, when a region of interest for the DM has been identified.

- Following directional searches, the DM can choose the objective function he wants to improve at each moment, having also the possibility of imposing constraints on the objective function values. These constraints may be revised whenever the DM wants, either by relaxing or tightening the bounds. This is far more flexible than other procedures, such as the STEM method in which the DM specifies a relaxation quantity for one objective function in order to improve the other objectives, but has not the possibility of selecting a criterion he would desire to be privileged in the following computation(s).

- If a local analysis is made (which may be useful in a final phase of the decision process) the DM can easily conclude whether a region of interest is completely explored or not.

- Trajectories of successive efficient solutions obtained throughout directional searches, combined with the analysis of their stability regions, can give a good perception of the geometry of the efficient solution set. Although stability regions concern the change of the reference point in the scalarizing program, they also provide stability degrees 
for the efficient solutions with respect to the improvement of a given objective function. Those "consecutive" efficient solutions may present similar values for all the objective functions or very different values in one (or more) objective(s). The DM can thus perceive these asymmetries.

\section{Acknowledgment}

This work was supported in part by the Foundation for Science and Technology (FCT) under Grants POCTI/ESE/ 38422/2001 and POSI/37346/SRI/2001.

\section{References}

[1] Fotuhi-Firuzabad M, Billinton R. Impact of load management on composite system reliability evaluation short-term operating benefits. IEEE Transactions on Power Systems 2000;15(2):858-64.

[2] Heffner G, Goldman C. Demand responsive programs—an emerging resource for competitive electricity markets?. Proceedings of the international energy program evaluation conference (2001 IEPEC), August 2001.

[3] Hirst E, Kirby B. Retail-load participation in competitive wholesale electricity markets. Edison Electric Institute, January 2001.

[4] Bowman VJ. On the relationship of the Tchebycheff norm and the efficient frontier of multiple-criteria objectives. In: Thiriez H, Zionts S, editors. Multiple criteria decision making. Lecture notes in economics and mathematical systems, vol. 130. Berlin: Springer; 1976. p. 76-86.

[5] Wierzbicki A. The use of reference objectives in multiobjective optimization. In: Fandel G, Gal T, editors. Multiple criteria decision making, theory and application. Lecture notes in economics and mathematical systems, vol. 177. Berlin: Springer; 1980. p. $468-86$.

[6] Durso A. An interactive combined branch-and-bound/Tchebycheff algorithm for multiple criteria. In: Goicoechea A, Duckstein L, Zionts S, editors. Multiple criteria decision making, proceedings of the ninth conference, Springer; 1992. p. 107-22.

[7] Narula SC, Vassilev V. An interactive algorithm for solving multiple objective integer linear programming problems. European Journal of Operational Research 1994;79:443-50.

[8] Karaivanova J, Korhonen P, Narula S, Wallenius J, Vassilev V. A reference direction approach to multiple objective integer linear programming. European Journal of Operational Research 1995;81:176-87.

[9] Alves MJ, Climaco J. An interactive reference point approach for multiobjective mixed-integer programming using branch and bound. European Journal of Operational Research 2000;124:478-94.

[10] Wierzbicki AP. Reference points in vector optimization and decision support. Interim Report, IR-98-017, IIASA, Laxenburg, Austria, 1998.

[11] Alves MJ, Clímaco J. Indifference sets of reference points in multiobjective integer linear programming. Journal of Multi-Criteria Decision Analysis 2001;10:177-89.

[12] Jorge H, Antunes CH, Martins AG. A multiple objective decision support model for the selection of remote load control strategies. IEEE Transactions on Power Systems 2000;5:865-72.

[13] Gomes A, Martins AG. Electric load modeling and simulation for assessing DSM actions. Proceedings of III international congress of environmental and technological innovations, vol. 3, Caracas, Venezuela, 1995. p. 449-54.

[14] Benayoun R, Montgolfier J, Tergny J, Larichev O. Linear programming with multiple objective functions: step method (STEM). Mathematical Programming 1971;1:366-75. 